\title{
Slow Cortical Potential Biofeedback and the Startle Reflex ${ }^{1}$
}

\author{
Stuart Brody, Harald Rau, Fabiola Köhler, Harald Schupp, \\ Werner Lutzenberger \\ University of Tübingen
}

Niels Birbaumer

University of Tübingen and Universita Degli Studi, Padova

The negativity of slow cortical potentials (SCP) of the surface EEG is a measure of brain excitability, correlating with motor and cognitive preparation. Selfcontrol of SCP positivity has been shown to reduce seizure activity. Following $S C P$ biofeedback from a central EEG electrode position, subjects gained bidirectional control over their SCP. The current study used a modified feedback methodology, and found a positive relationship between negativity and magnitude of EMG startle response (a measure of cortical and subcortical arousal, particularly aversive response disposition). Greater success in SCP differentiation was associated with self-report of less relaxation during negativity training.

Descriptor Key Words: biofeedback; slow cortical potentials; threshold regulation of EEG; startle probe.

In approximately $30 \%$ of patients with seizure disorders, their disease is not controllable by medication, and even those so controlled may suffer from side effects. Hence, the addition of a nonpharmacological intervention to the armamentarium against epilepsy would be valuable. Epilepsy is associated with overexcitability of cortical networks, resulting in negative cortical dc EEG shifts. Prior studies have demonstrated the efficacy of slow

${ }^{1}$ This research was supported by the Deutsche Forschungsgemeinschaft under grant No. SFB 307.

${ }^{2}$ Address all correspondence to Dr. Harald Rau, University of Tübingen, Institute of Medical Psychology and Behavioral Neurobiology, Gartenstrasse 29, D-72074 Tübingen, Germany. 
cortical potential (SCP) biofeedback in the treatment of epilepsy (Rockstroh et al., 1993), and the primary purpose of the present study is presentation of a modification of the SCP biofeedback technology. A second purpose of the study is the examination of changes in cortical excitability following SCP feedback, as measured by the startle reflex probe.

\section{SLOW CORTICAL POTENTIALS}

Slow cortical negativities and positivities (minimal duration 200-300 $\mathrm{ms}$ ) have their origin in the upper cortical apical dendritic layer (Birbaumer et al., 1990). Synchronous depolarization of the apical dendrites results in an electrically negative potential at the cortical surface and the scalp. The depolarization may be caused by intracortical afferents (about $60 \%$ of all fibers) or by unspecific thalamocortical afferents (about $30 \%$ of the incoming fibers). The origin of positive slow potentials is more difficult to explain and may result from less afferent excitatory influx. In addition, actual firing of the pyramidal neuron at the level of the basal dendrites and axons seems to be the main reason for surface cortical positivities. Birbaumer et al. (1990) have shown in a series of experiments that cortical negativity indicates a condition of mobilization ("cortical potentiality"), while positivity covaries with disfacilitation of cortical networks and behavior even during actual firing of layer III to V neurons. While there is no one-to-one correspondence between a specific neurochemical mechanism and SCP generation, there is some evidence (Timsit-Berthier et al., 1986; Rockstroh et al., 1990a) that acetylcholine is related to at least the earlier components of the SCP. Since the catecholamines inhibit the inhibitory effect of GABA, increased catecholaminergic activity is also associated with enhanced negativity.

Uncontrolled cortical overactivity may result in seizures. Speckmann and Elger (1987) reported that prior to seizures and their stereotypic spike and wave EEG waveforms, an SCP negative shift of approximately $100 \mu \mathrm{V}$ is observed. Although changes in cortical excitability are of briefer (generally less than $6 \mathrm{~s}$ ) duration than overall activation level, the finding of Speckmann and Elgar implies that increased cortical excitation increases the likelihood of a seizure. In humans, Elbert et al. (1990) developed a training procedure that allows patients to manipulate their cortical excitability. SCP biofeedback assisted subjects to gain control over negativity and positivity, increasing awareness of processes related to the development of a seizure, and decreasing seizure frequency and intensity (Rockstroh et al., 1993; Birbaumer et al., 1991).

Epileptics as well as normals have been able to achieve about 10-20 $\mu \mathrm{V}$ control of SCP (Birbaumer et al., 1991). Normals needed as few as 
two sessions (Roberts et al., 1989), but epileptics required 28 sessions to attain this control, and for all subjects it was generally easier to enhance negativity. Gaining mastery over positive shifts has been more difficult. Some epileptics experienced dramatic reductions in seizure frequency from positivity training, but others showed little or no change. One goal of the present study was to develop a biofeedback technique that would both enhance SCP control for a larger percentage of subjects and speed learning.

The new design used a continuous shaping feedback algorithm which initially reinforced small EEG shifts and led the subject to build upon them. Alternate trials of negativity and positivity were required. The previous design (Elbert et al., 1980, 1990) had a fixed trial length, while the new approach is more individualized. Successful trials were followed by a change of condition (from positivity training and its associated feedback graphics to negativity, or vice versa) contingent upon a spontaneous shift in the opposite direction.

\section{STARTLE PROBE}

The behavioral impact of SCP changes has been probed by reaction time and other measures (Rockstroh et al., 1990a). In the current study, we used the startle probe. An advantage of the startle probe might be that there is little competition with the primary biofeedback task, because startle stimuli are not followed by extensive processing (Anthony, 1985).

The startle reflex is elicited by the presentation of sudden and intense stimuli. The reflex is a whole-body flexor response and the eye blink is commonly used to measure the startle response (Anthony, 1985). Although the startle reflex is thought to be a stereotyped response, it shows considerable plasticity and can be used to index cortico-subcortical excitation (Schupp, Lutzenberger, Rau, \& Birbaumer, in press), particularly in the form of aversive response disposition (Lang et al., 1990).

To investigate cortical excitability during negative shifts (and correspondingly, decreased excitability during positivity), we elicited the startle reflex at different levels of negativity. We predicted larger startle magnitude during periods of increased negativity.

\section{METHODS}

\section{Subjects}

Ten student volunteers ( 4 male and 6 female), aged 19 to 29 (mean 24.7, $S D$ 3.6) served as subjects. Exclusion criteria included neurological 
or cardiovascular disease. Subjects were informed that they were free to terminate participation at any time without negative consequences.

\section{Procedure}

Four sessions were held on separate days. On each day, there were two series consisting of two 5-min feedback blocks followed by one transfer block. Between blocks, subjects had the option of resting before proceeding.

Televised feedback of the slow potentials consisted of a diamond which moved for up to $10 \mathrm{~s}$ toward an arrow contingent on the SCP shift required at the moment, or backward when performing poorly. The diamond became a smile face symbol when the goal was reached (see Figure 1). Failure to reach it within $10 \mathrm{~s}$ resulted in a reversal of condition (polarity and diamond movement). The arrow pointed (and was located on the) right for negativity and left for positivity, and its position indicated the level of $\mathrm{SCP}$ shift required. Because eye movements produce what might be inter-

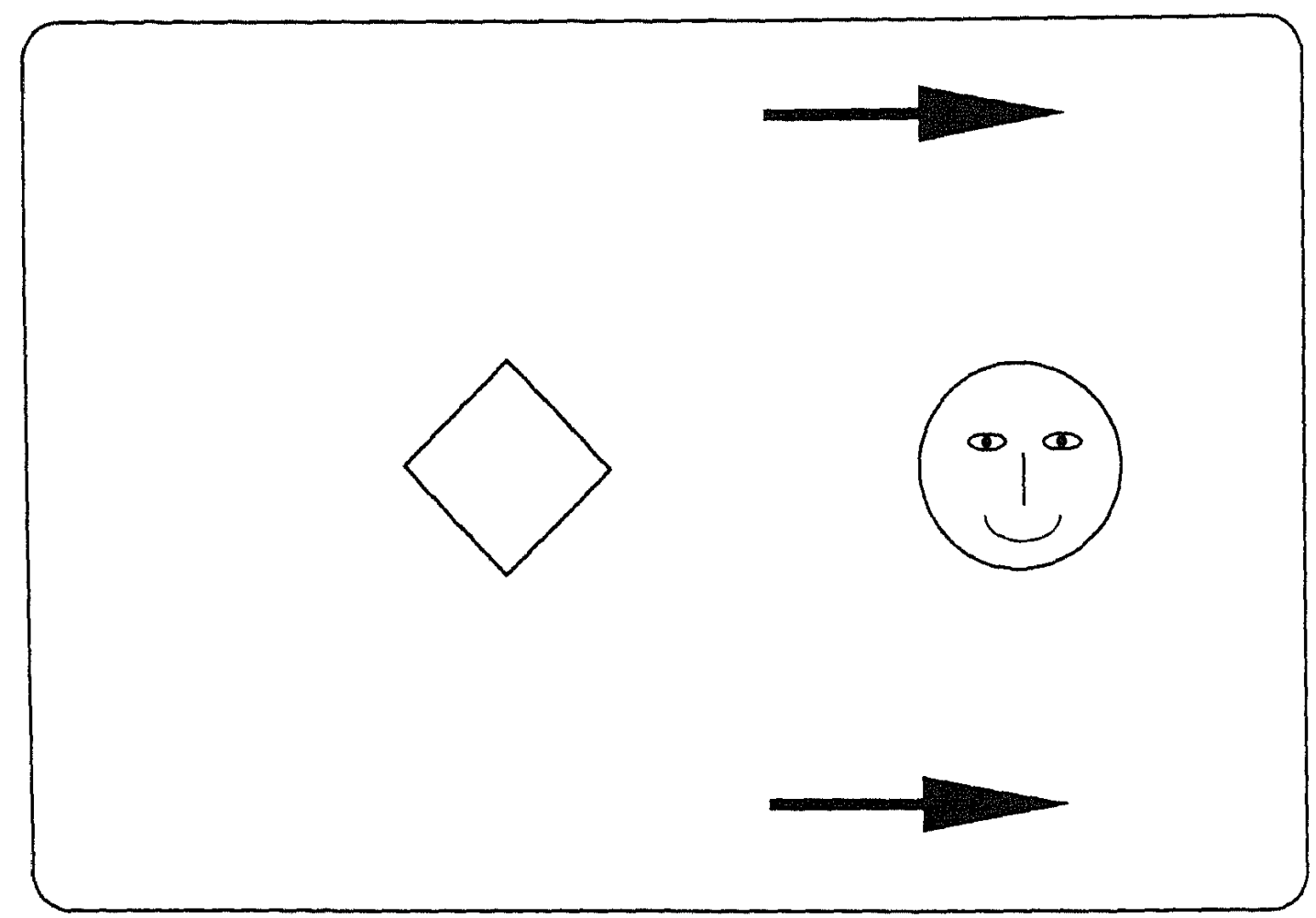

Fig. 1. Feedback image. The diamond moves horizontally as a function of $\mathrm{Cz}$ (linked ears reference) dc shifts. The arrows indicate the direction required (left increased negativity and right positivity). Eye movements in the supporting direction were compensated for by the computer moving the arrows away from the center and thus making the criterion for a successful trial (the diamond reaching the arrows) more difficult. If the criterion was met, the diamond changed to a smiling face, and arrows changed their position and direction, beginning a new trial. 
preted as SCP shifts (Rockstroh et al., 1990), the arrow was displaced away from success when an eye movement simulating the shift was detected (and the corrected value was used in analyses). Thus, eye movement could only work against goal attainment. Visual feedback was a linear function of the 500 -ms moving average EEG (based on 100-ms steps). Feedback was given from the $\mathrm{Cz}$ electrode.

The first goal in the shaping sequence was producing a change of 5 $\mu \mathrm{V}$ in the desired direction (positivity or negativity). Goal attainment within $10 \mathrm{~s}$ led to a new goal, the absolute value of which equaled the maximum value achieved in the most recent effort plus $3 \mu \mathrm{V}$. Goal attainment was followed by a change of condition. Failure to reach the goal within the time limit led to a goal that equaled the maximum achieved minus $2 \mu \mathrm{V}$. These goal-setting rules were subject to the restriction that the difference between positivity and negativity goals be no less than $4 \mu \mathrm{V}$.

Transfer trials (testing the ability of the subjects to exert control over slow cortical potentials in the absence of feedback) consisted of two blocks of $5 \mathrm{~min}$ each. Subjects were shown the same arrow (without the diamond) used during feedback, and the duration and position (goal) were equal to the mean values obtained during the most recent feedback trial.

Subjects received a total of 28 acoustic startle probes during the third and fourth SCP training sessions. The probes were delivered during each of the second feedback blocks within a series. A minimum of $30 \mathrm{~s}$ passed between probes, and probe timing alternated between positivity, negativity, and neutral conditions according to the following sequences: Neutral Neg Pos Neutral Pos Neg, or Neutral Pos Neg Neutral Neg Pos. If no shift to the next expected condition occurred within the next $30 \mathrm{~s}$, a probe was given anyway.

A questionnaire was given to ascertain which strategies made for success in each condition of the feedback paradigm. Subjects rated relaxation, pleasant feelings, and body movement on a five-point scale after the final session.

\section{Data Recording}

EEG was recorded from $\mathrm{Cz}, \mathrm{Fz}$, and $\mathrm{Pz}$, referenced to linked earlobes, using $\mathrm{Ag} / \mathrm{AgCl}$ electrodes. Electrode impedance was $5 \mathrm{k} \Omega$ or less, and a time constant of $4 \mathrm{~s}$ was used. A sampling rate of $100 \mathrm{~Hz}$, and a high-frequency cutoff of $35 \mathrm{~Hz}$ were used. Vertical eye movements were recorded and amplified similarly.

The EMG of the right orbicularis oculi muscle was recorded using Beckmann surface minielectrodes. The signal was amplified by a Nihon 
Kohden amplifier with a $70-500-\mathrm{Hz}$ bandpass. The recording interval began with the noise onset and continued for $700 \mathrm{~ms}$. A sampling rate of $2 \mathrm{kHz}$ was used.

Startle probe intensity was individually adjusted during baseline measurements between 90 and $100 \mathrm{~dB}$ (A-weighted, $0 \mathrm{~dB}$ referenced to $2.0 \times$ $10^{-4} \mu \mathrm{bar}$ ) of pseudorandomized white noise produced by a D/A converter operating at $20 \mathrm{kHz}$ (50 ms duration, delivered through headphones) to elicit a clear startle reflex.

\section{Data Analysis}

EMG recordings were analyzed off-line by the "denosing in quadrature" procedure described by Fridlund and Cacioppo (1975). EMG magnitude was calculated as the logarithm of the sum of squares in a window 10-70 ms following startle onset. Trials with an unstable baseline in the 20 -ms window after the startling noise onset were eliminated because that noise would likely contaminate the dependent variable.

For the analysis of SCP control, only results from the transfer trials were used. A repeated measures ANOVA was performed, with the independent variables Electrode $(\mathrm{Fz}, \mathrm{Cz}$, and $\mathrm{Pz}$ ), Session, Condition (positivity or negativity reinforced), and Series (first or second within a session), and the dependent variable of cortical negativity $(\mu \mathrm{V})$.

Analysis of the relation between SCP and cortical arousal as measured by the startle probe was accomplished by means of a repeated measures ANOVA with the independent variable of Startle magnitude quartile (each quartile containing 5 probes) and the dependent variable of cortical negativity $(\mu \mathrm{V})$.

Hunyh-Feldt epsilon (H-F) corrected $p$ values are reported for repeated measures factors with more than two levels. Unadjusted degrees of freedom are reported. The alpha significance level was set at .05.

\section{RESULTS}

\section{Slow Cortical Potentials}

A significant Electrode effect was found $(F(2,18)=8.1, p=.0031)$ with post hoc tests indicating a significant difference between $\mathrm{Fz}$ and $\mathrm{Cz}$ (post hoc $F=14.4, p=.0013$ ), as well as $\mathrm{Fz}$ and $\mathrm{Pz}$ (post hoc $F=9.4$, $p=.0078$ ), but not $\mathrm{Cz}$ and $\mathrm{Pz}$ (post hoc $F=.5$ ). This effect is portrayed in Figure 2, which is averaged across both series of the first two sessions 


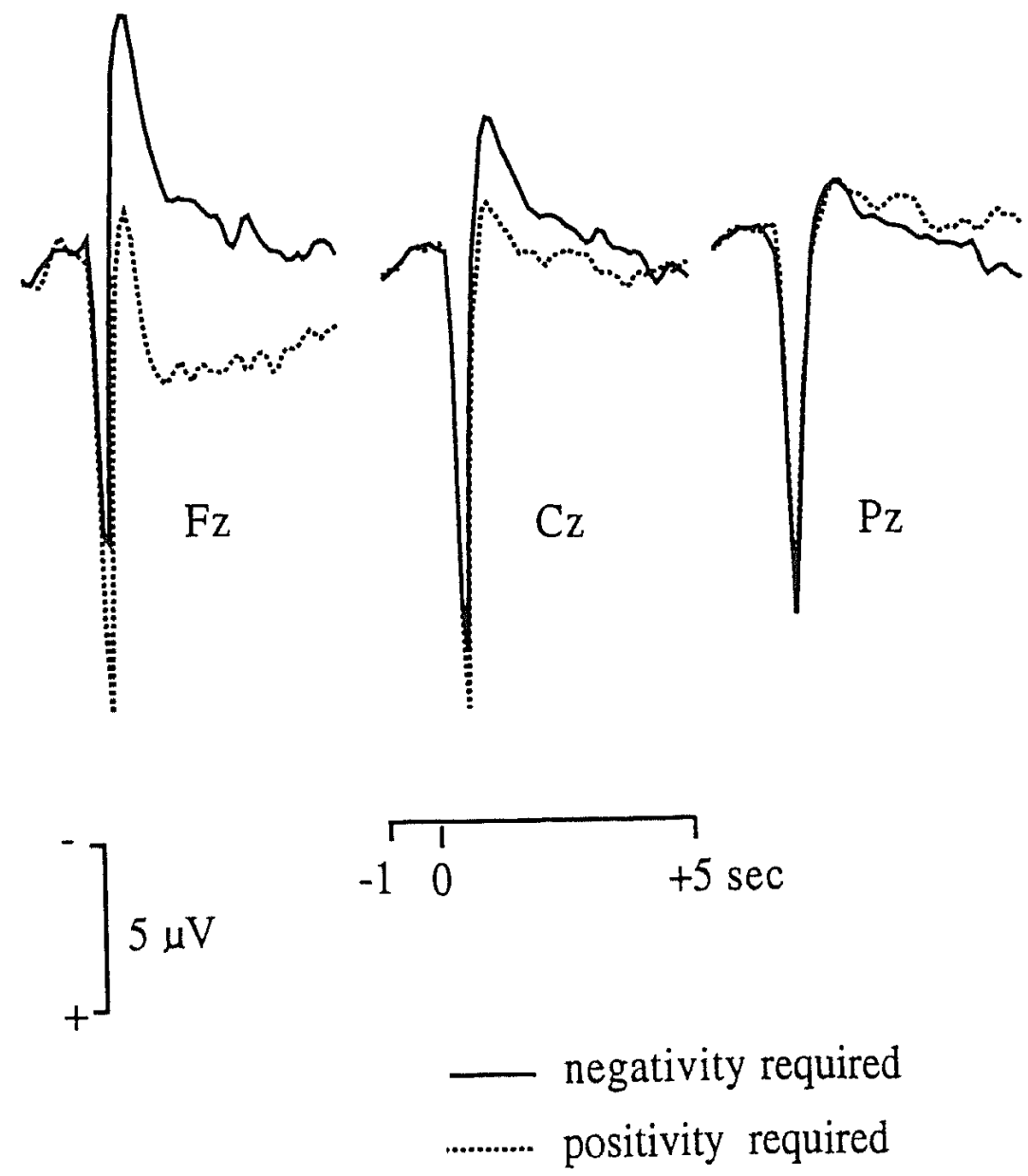

Fig. 2. Differentiation of SCP at electrodes $\mathrm{Fz}, \mathrm{Cz}$, and Pz. Solid line: Negativity required. Broken line: Positivity required. The higher-frequency, lower-amplitude transient potentials (about 1 $\mathrm{Hz},<1 \mu \mathrm{V})$ are noise, but are noticeable because of the small sample size.

(the latter two sessions were not included because of the startle probes). Neither Session nor Series was a significant factor $(F$ 's $=.8)$. Condition was significant in the labeled direction $(F(1,9)=6.9, p=.028)$.

The significant Electrode-Condition interaction $(F(2,18)=11.1, p$ $=.004$ ) was located largely at the contrast of negative and positive conditions at $\mathrm{Fz}$ (post hoc $F=35.7, p=.0002$ ). Table I lists the means and SDs of negativity for the six combinations.

Series-Condition showed a significant interaction $(F(1,9)=5.6, p=$ $.042)$ in the direction of less differentiation with later series.

Electrode-Series, Session-Series, Session-Condition, Electrode-SessionCondition, Electrode-Session-Series-Condition interactions were not significant (all $F$ 's less than 1 ). Electrodes-Session-Series $(F(6,54)=2.0, p=$ $.10)$, Session-Series-Condition $(F(3,27)=1.5, p=.24)$, Electrode-Series- 
Table I. Means of Electrode-Condition Interactions

\begin{tabular}{lccc}
\hline Electrode-Condition & Count & Mean & $\begin{array}{c}\text { Standard } \\
\text { deviation }\end{array}$ \\
\hline Fz-Neg & 80 & -1.81 & 4.98 \\
Fz-Pos & 80 & 2.37 & 6.08 \\
Cz-Neg & 80 & -1.44 & 4.44 \\
Cz-Pos & 80 & -.41 & 4.93 \\
Pz-Neg & 80 & -.51 & 3.85 \\
Pz-Pos & 80 & -.89 & 3.97 \\
\hline
\end{tabular}

Condition $(F(2,18)=1.6, p=.23)$ and Electrode-Session $(F(6,54)=2.3$, $p=.06)$ interactions all missed significance.

When the frequency distribution of each subject's mean negativity was examined, it was found that only 2 of 10 subjects achieved at least a $5-\mu \mathrm{V}$ negativity shift in the first session ( 3 in the second), and only 4 met the $5-\mu \mathrm{V}$ criterion for positivity in the first session ( 2 in the second). The third and fourth sessions were not examined in this manner, because of the startle probes during those sessions.

The EOG differentiation was not significant $(F(1,9)=3.8 ; p=.084)$ and trended in the direction opposite that of the significant $E E G$ findings.

\section{Startle Probe}

Negativity was significantly related to startle magnitude $(F(1,9)=3.2$, $p=.05)$. As shown in Figure 3, the fourth Quartile of startle magnitude showed a significantly greater (post hoc $F=8.8, p=.01$ ) positivity than the first quartile (mean first quartile $=-1.8, S D 12.1$; mean fourth quartile $=9.9, S D=12.9)$. A significant linear post hoc effect was found $(F=9.4$; $p=.008$ ) for the startle quartiles. Quartiles were used because signal-tonoise ratios are so poor in EEG recording of SCP (and many other values) that a useful signal is obtained only after averaging several trials. There was no significant difference between electrodes by condition $(F=.6)$.

\section{Questionnaire}

The differentiation between positivity and negativity at $\mathrm{Fz}$ was correlated with subjects' ratings of their mental state and motor behavior during each of the two conditions. The questionnaire results indicated that less pleasant feelings were more associated with negativity strategy than with 


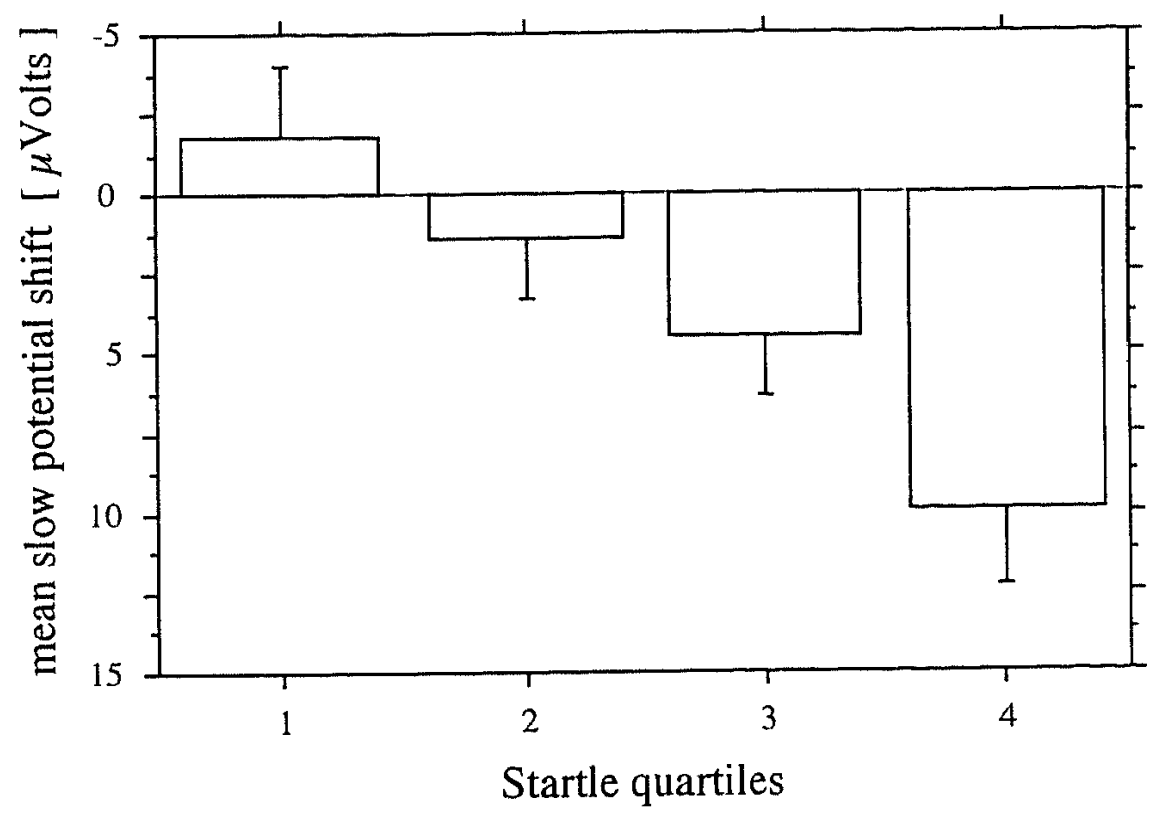

Fig. 3. Startle magnitude quartiles (first quartile is strongest startle) and SCP. The error bars are one standard deviation.

positivity strategy $(t(9)=2.45, p=.037)$. Body movement and relaxation did not reach significance.

However, when the correlation between positivity and negativity differentiation and strategy was examined, a strong association $(r(8)=.71, p$ $=.023$ ) between that differentiation and less relaxation during negativity training was found. Body movement and pleasant feelings during negativity training, as well as all three strategies during positivity training were not significantly related to differentiation.

We noted a trend toward women exhibiting greater differentiation than men $(t(8)=1.93, p=.09$ ), and then found that women reported less relaxation during negativity $(t(8)=3.65, p=.0065)$ than men.

\section{DISCUSSION}

The results indicate that some individuals have a significant ability to learn differential SCP shifts, and that this learning occurs within the first session, with no additional gain in three subsequent sessions. These bidirectional effects appear most prominently in the frontal region, but there is some unidirectional (negativity only) central effect as well.

Subjects appear to fatigue quickly, as indicated by the Series-Condition interaction. The insignificance (with adequate statistical power) of Ses- 
sion-Condition suggests that within the limited framework of four sessions, there is no additional learning occurring after the first session.

The percentage of strong responders within the current feedback parameters appears somewhat limited, and this factor may also contribute to the finding of no incremental gain with additional sessions. It should be noted that although the magnitude of control obtained is small, exercising control early in an epileptic neurophysiological sequence may be sufficient to abort the seizure. Further research may investigate whether lengthier training or modification of other feedback features (such as using the frontal electrode as the feedback electrode) will enlarge the population of responders.

The startle data support the hypothesis that relative cortical positivity (or decreased negativity) is associated with diminished mobilization of cortical networks. Cortico-subcortical modulation of the startle reflex is possible via an anatomical connection from frontal regions terminating in the subcortical reticular nuclei subserving the organization of the startle reflex. In the animal literature this modulatory connection is inhibitory, while the second principal modulatory pathway originating in the amygdala reduces the firing thresholds of the reticular startle nuclei (Davis, 1992).

The questionnaire results suggest that successful SCP differentiation is associated with less relaxation during negativity feedback, supporting the characterization of negativity as a state of readiness. These data confirm earlier studies (e.g., Roberts et al., 1989). The trend toward women producing superior differentiation may be attributed to women in our sample avoiding relaxation during negativity training.

Other potential applications of SCP biofeedback include increasing negativity in disorders such as Attention Deficit Disorder (Rockstroh et al., 1990b) or some of the schizophrenias (Schneider et al., 1992).

\section{REFERENCES}

Anthony, B. J. (1985). In the blink of an eye: Implications of reflex modification for information processing. In P. K. Ackles, J. R. Jennings, \& M. G. H. Coles (Eds.), Advances in psychophysiology (Vol. 1, pp. 167-218). Greenwich, CT: JAI Press.

Birbaumer, N., Elbert, T., Canavan, A. G. M., \& Rockstroh, B. (1990). Slow potentials of the cerebral cortex and behavior. Physiological Reviews, 70(1), 1-41.

Birbaumer, N., Elbert, T., Rockstroh, B., Daum, I., Wolf, P., \& Canavan, A. (1991). Clinical psychological treatment of epileptic seizures: A controlled study. In A. Ehlers et al. (Eds.), Perspectives and promises of clinical psychology. New York: Plenum Press.

Davis, M. (1992). The role of the amygdala in fear-potentiated startle: Implications for animal models of anxiety. Trends in Pharmacological Science, 13, 35-41.

Elbert, T., Rockstroh, B., Canavan, A., Birbaumer, N., Lutzenberger, W., von Bulow, I., \& Linden, A. (1990). Self-regulation of slow cortical potentials and its role in 
epileptogenesis. In J. Carlson \& R. Seifert (Eds.), Biobehavioral self-regulation and health. New York: Plenum Press.

Elbert, T., Rockstroh, B., Lutzenberger, W., \& Birbaumer, N. (1980). Biofeedback of slow cortical potentials. Electroencephalography and Clinical Neurophysiology, 48, 293-301.

Fridlund, A. J., \& Cacioppo, J. T. (1975). Guidelines for electromyographic research. Psychophysiology, 12(3), 238-248.

Lang, P. J., Bradley, M. M., \& Cutbert, B. N. (1990). Emotion, attention, and the startle reflex. Psychological Review, 97, 377-398.

Roberts, L., Rockstroh, B., Lutzenberger, W., Elbert, T., \& Birbaumer, N. (1989). Self-reports during feedback regulation of slow cortical potentials. Psychophysiology, 26, 392-403.

Rockstroh, B., Elbert, T., Birbaumer, N., Wolf, P., Düchting-Röth, A., Reker, M., Daum, I., Lutzenberger, W., \& Dichgans, J. (1993). Cortical self-regulation in patients with epilepsies. Epilepsy Research, 14, 63-72.

Rockstroh, B., Elbert, T., Canavan, A. G. M., Lutzenberger, W., \& Birbaumer, N. (1990). Slow cortical potentials and behaviour: 2nd ed. Munich: Urban \& Schwarzenberg.

Rockstroh, B., Elbert, T., Lutzenberger, W., \& Birbaumer, N. (1990). Biofeedback: Evaluation and therapy in children with attentional dysfunctions. In $H$. Rotherberger (Ed.) Brain and behavior in child psychiatry (pp. 345-357). Berlin: Springer.

Schneider, F., Rockstroh, B., Heimann, H., Lutzenberger, W., Mattes, R., Elbert, T., Birbaumer, N., \& Bartels, M. (1992). Self-regulation of slow cortical potentials in psychiatric patients: Schizophrenia. Biofeedback and Self-Regulation, 17(4), 277-292.

Schupp, H., Lutzenberger, W., Rau, H., \& Birbaumer, N. (in press). Positive shifts of event-related potentials: A state of cortical disfacilitation as reflected by the startle reflex probe. Electroencephalography and Clinical Neurophysiology.

Speckmann, E. J., \& Elger, J. (1987). Introduction to the neurophysiological basis of EEG and DC potentials. In E. Niedermeyer \& F. Lopes da Silva (Eds.), Electroencephalography, 2nd ed. (pp. 1-14). Baltimore: Urban \& Schwarzenberg.

Timsit-Berthier, M., Mantanus, H., Marissaux, P., Ansseau, M., Doumont, A., Geenen, V., \& Legros, J. (1986). CNV and dopamine receptor reactivity: Correlations with the apomorphine test. In W. C. McCallum, R. Zappoli, \& F. Denoth (Eds.) Cerebral psychophysiology: Studies in event-related potentials (pp. 403-405). Amsterdam: Elsevier. 